\title{
To the Question of Multi-Criteria Optimization of Aircraft Components in Order to Optimize its Life Cycle
}

\author{
Sergey Alekseevich Serebryansky*, Alexander Vladimirovich Barabanov
}

Department Aircraft Design and Certification, Moscow Aviation Institute (National Research University), Moscow, 125993, Russia

\begin{tabular}{l} 
A R T I C L E I N F O \\
\hline Article history: \\
Received: 20 August, 2020 \\
Accepted: 21 October, 2020 \\
Online: 20 November, 2020 \\
\hline Keywords: \\
Complex Methodology \\
Optimization of The Design Cycle \\
Aerodynamic Drag \\
Rational Parameters \\
Nose of The Aircraft \\
\hline
\end{tabular}

\begin{abstract}
A B S T R A C T
The paper examines the impact on the product life cycle of reducing the duration of the development stages of aviation technology through the use of multi-criteria optimization. The subject of the study is the nose of a supersonic aircraft and the process of linking it. This example describes in detail the application of a comprehensive design methodology for aircraft components using top-level criteria as a way to significantly reduce the development time and the risks of obtaining negative test results when entering a full-scale sample.
\end{abstract}

\section{Introduction}

The great Russian aircraft designer Pavel Osipovich Sukhoi said: "We must not stop at what we have achieved, but must constantly move forward. After all, what was new and advanced yesterday will only be satisfactory tomorrow." This statement is still relevant until now because to take leadership positions in aviation, engineering, Economics, and business, you must constantly work ahead of time and your competitors. The current trend in the field of mechanical engineering is a significant (from $10-15$ to 5-8 years) reduction in the development time of new aircraft systems due to the use of mathematical modeling, high unification and the use of scientific and technical reserve. The use of complex design techniques is one of the elements based on scientific and technical groundwork and allows you to reduce the design time.

\section{Aircraft product life cycle}

Within the lifecycle of the aircraft as a product, the aircraft goes through the following stages (gates):

Figure 1 shows the typical life cycle of an aircraft product, which is divided into internal and external design stages. The focus of the current work is optimization of internal design stages, so we will analyze them in more detail.

It is worth noting that key technical decisions are made at the stages of the preliminary project and preliminary technical design.

\footnotetext{
*Corresponding Author: Sergey Alekseevich Serebryansky, s-s-alex@mail.ru
}

The work is carried out by a relatively small number of OKB employees, the minimum amount of financial resources from the project budget is used, and the maximum number of technical decisions is made as shown in Figure 2. According to the results of these stages, the appearance of the designed aircraft is fixed.

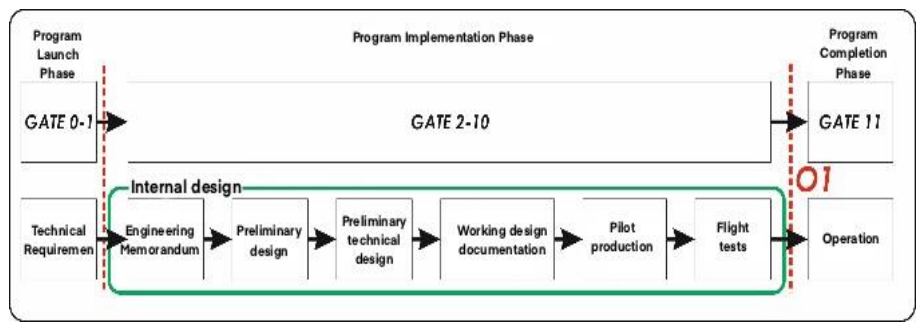

Figure 1: The typical lifecycle of the aviation system

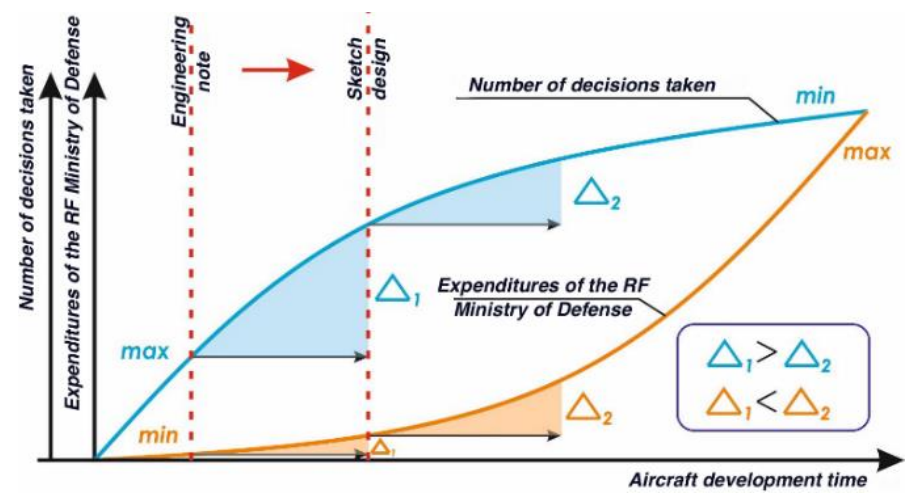

Figure 2: Dependence of the number of decisions made and the customer's costs on the project implementation time 


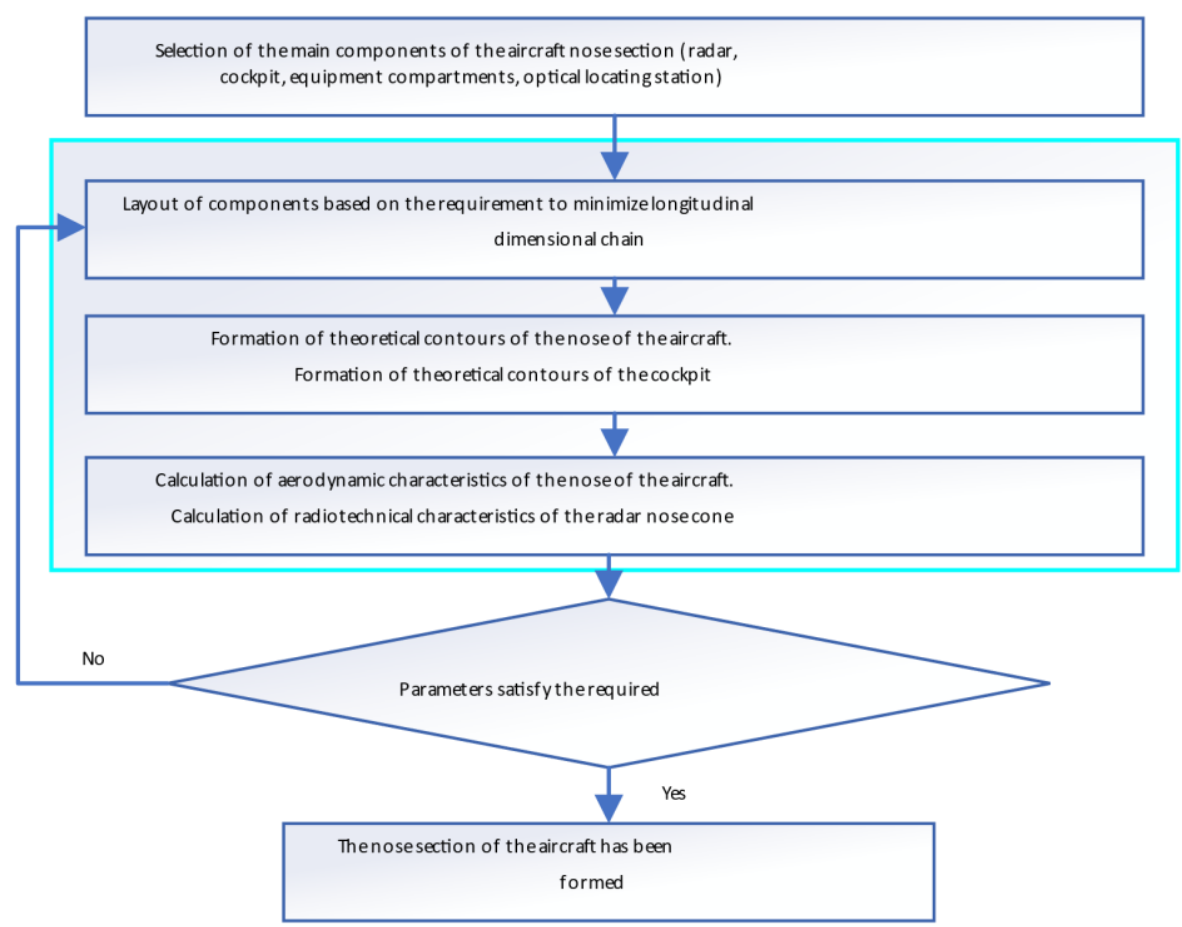

Figure 3: Typical algorithm for developing the nose of the aircraft

Thus, the stages of project initiation are the most effective in terms of cost and number of technical decisions:

$$
\left.\begin{array}{c}
\Delta_{i}^{\text {expenses }} \\
\Delta_{i}^{\text {time }}
\end{array}\right\} \rightarrow \min (\text { opt })
$$

However, these stages have the greatest technical risks, and errors in the technical decisions made may become fatal for the further fate of the project.

As can be seen from Figure 2, the initial stages of project implementation are the most effective in terms of the ratio of the customer's costs to the number of decisions made. Simultaneously, the technical decisions taken at these stages determine the further technical appearance of the project and can significantly affect its results.

Figure 3 shows an algorithm for linking the nose of the aircraft, consisting of sequential operations and, in case of errors, having an iterative character.

To complete the formation of the theoretical contour of the head part of the aircraft it must validate the obtained electronic and aerodynamic characteristics of the selected form of the radio waves fairing requirements to ensure the characteristics of the radar. Meanwhile, the speed and range characteristics of the aircraft will depend on the aerodynamic characteristics in the future, and the potential capabilities of the locator will depend on the radio technology. Thus, such a seemingly insignificant thing as the shape of the nose of an aircraft can determine its key characteristics of combat use. Moreover, the shape of the nose part of the minimum resistance and the shape of the maximum radar-transparency of a thing are incompatible because the best radio-technical characteristics are usually of small elongation nosecone (the ratio of the square of the length of the nosecone to the area of the initial cross-section), such a shape allows you to ensure the uniformity of the distribution of the angles of incidence of the electromagnetic wave front on the surface of the nosecone wall, the best aerodynamic characteristics on the contrary have the body with maximum elongation, it allows reducing significantly the resistance at supersonic flight speeds, see Figure 4:
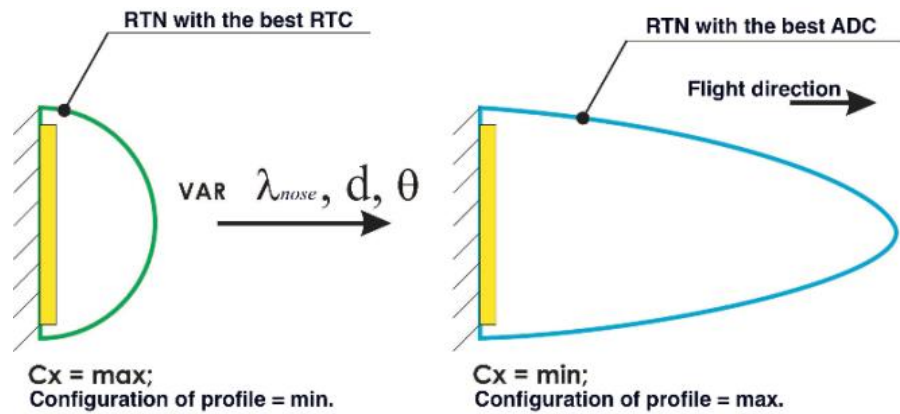

Figure 4: Comparison of nosecone shapes of the aircraft

From Figure 4 we can conclude that the final shape of the nosecone will be a compromise between the two shown in the figure. In this case, the wrong form at this stage may lead to the need to repeat the whole cycle of flight tests in case of its change after the construction of prototypes (stages of pilot production and flight tests).

\section{Optimization of the aircraft life cycle}

The scientific novelty of the work consists in carrying out multi-criteria optimization of the geometric parameters of the studied RPO using the Russian-made LOGOS software product and in terms of functions, which is an analog of the FLUENT product. The results of mathematical modeling and evaluation of aerodynamic characteristics have been validated with existing empirical methods, and the software product itself is widely used in Russian industrial projects. 


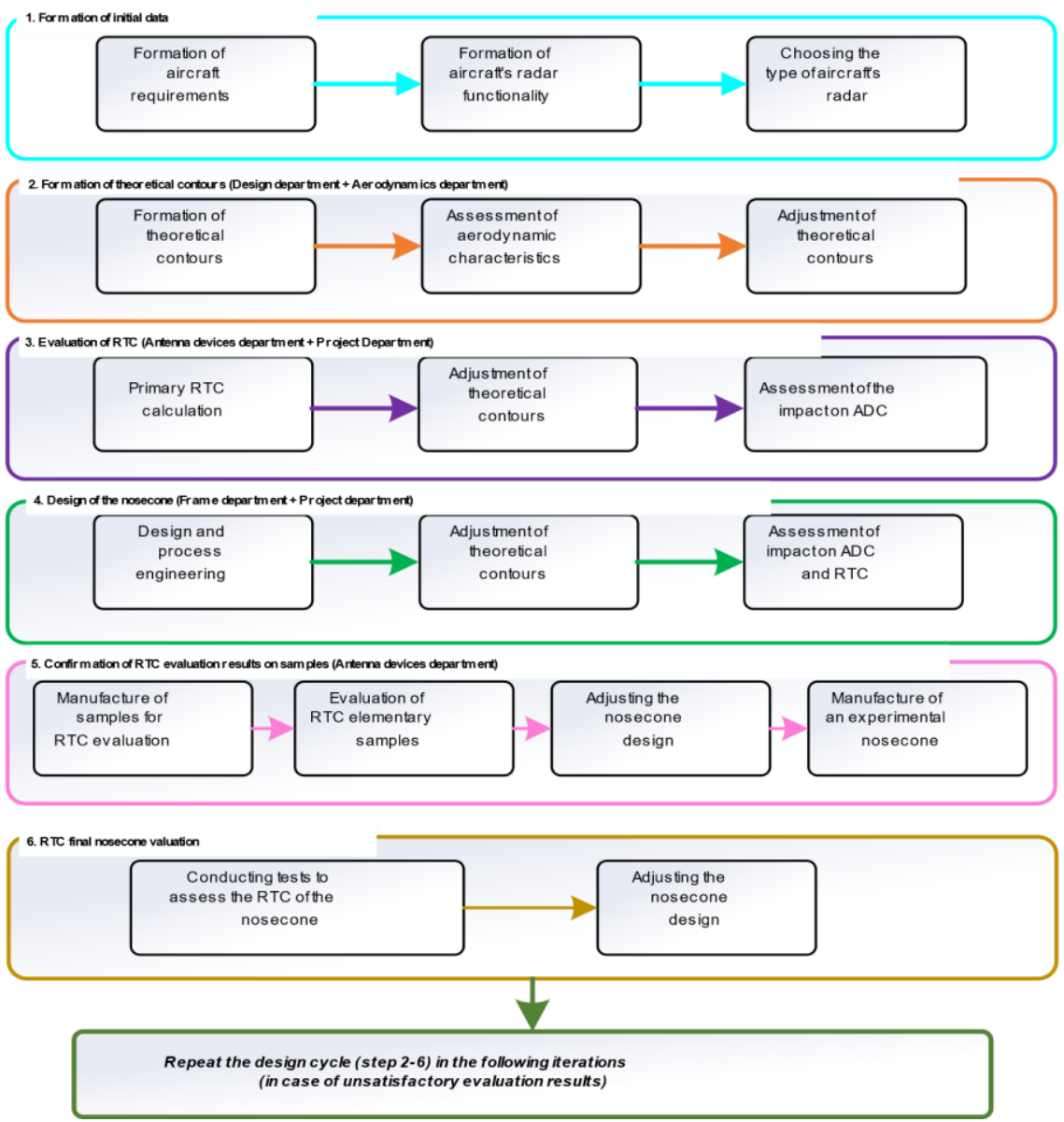

Figure 5: The existing algorithm for the development of the fairing

Reducing the timing of development work can be achieved by reducing the development and testing of the aircraft complex. Concurrently, correct and timely technical decisions made at the development stage will also reduce the time required for ground and flight tests. Let's look at an example of optimizing the development stages of a nose radio-transparent fairing (RPO). The current state of the RPO design algorithm is shown in Figure 5.

After analyzing Figure 4, we can conclude that the design of the radio-transparent fairing according to the existing methodology does not meet the requirements for the development of new aircraft systems. To meet the customer's requirements for the development of new AK in 5-8 years, it is necessary to significantly optimize this algorithm. To do this, you must: To optimize this algorithm, it is necessary:

1) In accordance with [1], [2] select a comprehensive criterion for evaluating the characteristics of RPO radar, both in terms of radio engineering (RTC) and aerodynamic (ADC) characteristics;

2) Form a research algorithm, in which to establish the relationship between the parameters of the shape of the fairing and its ADC and RTC.

3) Based on the results of the study, formulate technical recommendations for choosing rational parameters of the fairing shape, depending on the initial data and technical requirements.
As a complex criterion of the upper level (1), having analyzed the scientific and technical background in the field of research of radio technical characteristics (RTC) [3] and aerodynamic characteristics (ADC) [4], [5], [6], it is possible to take the ratio of the criterion of the first level of aerodynamic characteristics to the criterion of the first level of radio engineering characteristics:

$$
X^{*}=\operatorname{ArgMax}\left(\frac{\operatorname{ArgMax}(R T C)}{\operatorname{ArgMin}(A D C)}\right)
$$

In this case, we will choose as the criterion of the first level for RTC nosecone its transmission coefficient (TC) - a dimensionless value equal to the ratio of the radiation flow through the medium (nosecone) to the radiation flow to its surface. The first level criterion for ADC is its drag coefficient $(\mathrm{Cx})$, a dimensionless value reflecting how the body (nosecone) reacts with the environment (air).

According to this criterion, it is necessary to conduct research on possible options for the geometric appearance of the fairing and choose rational parameters that meet all the requirements. The algorithm for conducting these studies is shown in Figure 6.

Based on the results of the above studies, the method for selecting the geometric parameters of the fairing will allow optimizing the stages of RPO development. Adjusted according to the results of the optimization algorithm for the design of the fairing is presented in Figure 7. 


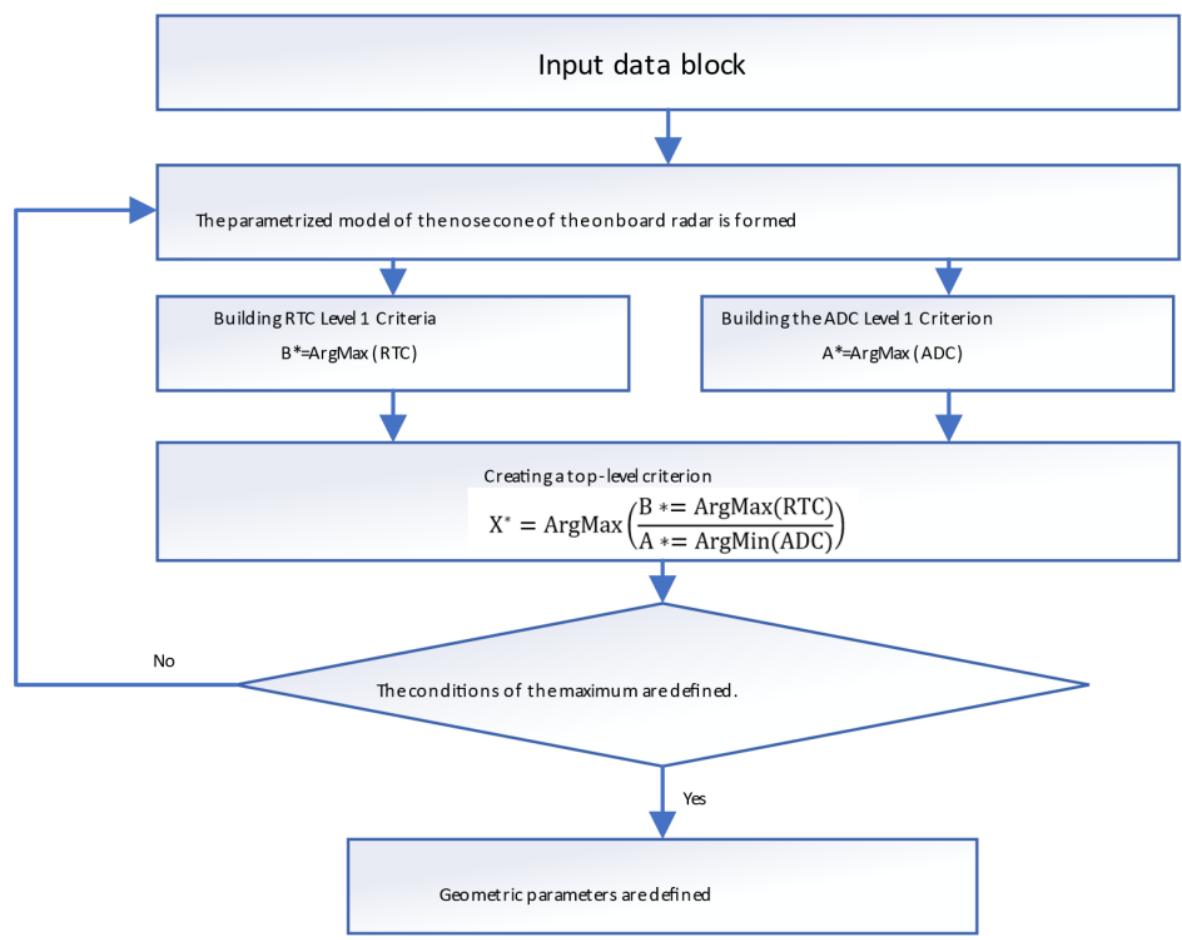

Figure 6: Algorithm for studying geometric parameters of RPO

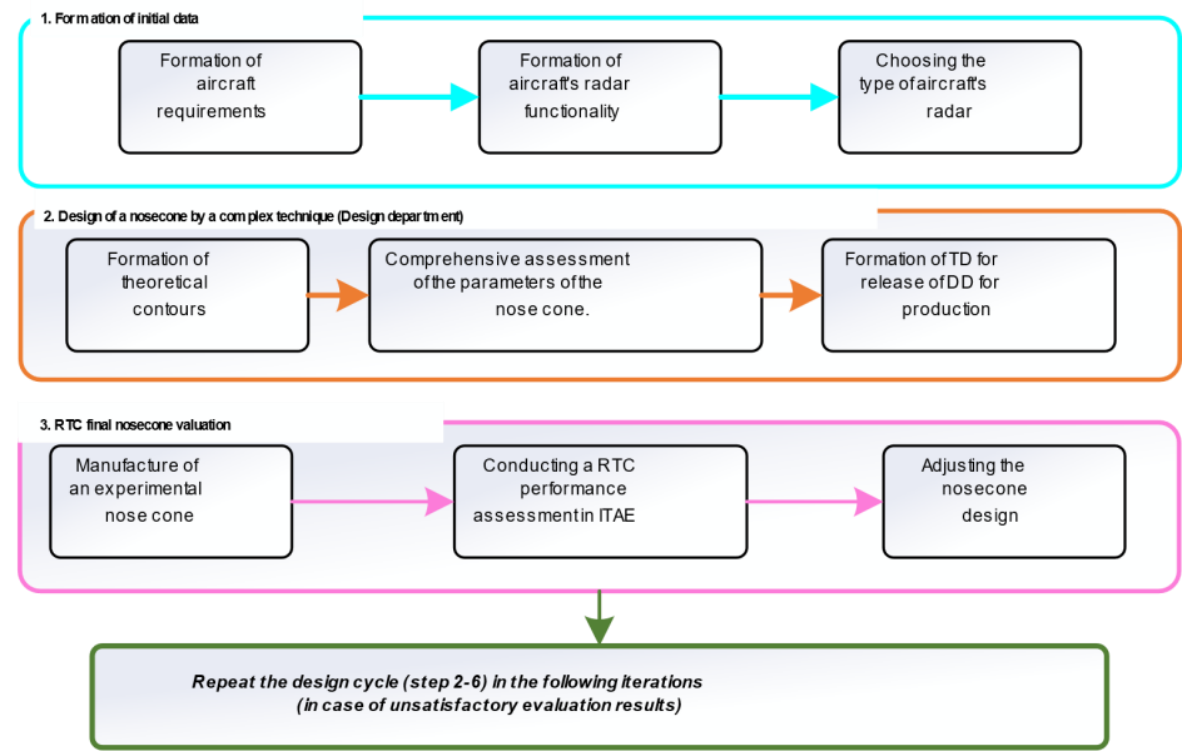

Figure 7: Adjusted the algorithm design of the fairing

Thus, the development of such methods for designing aircraft components can significantly reduce the number of design stages, as well as the involvement of company personnel, which in turn reduces the time and cost of product development.

\section{Forming an optimal fairing with minimal drag}

A typical example of multi-criteria optimization of an aircraft component to significantly reduce the product life cycle is the optimization of the geometric parameters of the radio-transparent fairing in order to obtain a body of minimal resistance. For this optimization, you must:

Firstly, to choose a complex criterion for evaluation of aerodynamic characteristics;
Secondly, to make up an algorithm for solving this problem and determine a method for evaluating aerodynamic characteristics that will have the required level of sensitivity to changes in key geometric parameters and the required accuracy;

Third, using the selected algorithm, to conduct research and, using a complex criterion, select rational geometrical parameters of the nose section of the aircraft for a given flight mode.

\subsection{Choosing a comprehensive criterion}

As noted earlier in this work, the issue of selecting rational geometric parameters for the nose section of the aircraft for the supersonic cruise mode is under consideration $M \sim 1.5$. To select the main criterion of aerodynamic characteristics of the nose 
section, it is necessary to analyze the existing aerodynamic characteristics of the aircraft components as a whole.

According to [7], there are three main criteria for the aerodynamic characteristics of an object: $C_{x}-$ aerodynamic resistance coefficient, $C_{y}-$ lifting factor, $\Delta m z_{0}-$ component contribution to the longitudinal static torque of the aircraft as a whole:

1) Coefficient $C_{\text {xnose }}$ as shown in Part 1. The introduction is one of the essential parts of the aerodynamic drag coefficient of the pressure of the aircraft as a whole $C_{x f p r e s s u r e}$ in particular for $M>1$.

2) In doing so $C_{\text {ynose }}$ does not make a significant contribution to is not more than $0.1 \%$ of the total $C_{y}$ as a whole.

3) It should be noted that the nose part of the aircraft can be shaped as a body rotation, and as a body of complex shape in the cross section. So for the body of rotation the contribution to $\Delta m z_{0}$ the aircraft as a whole tends to 0 , and for a complex body it requires deep analysis and has a significant impact due to the large removal of the aerodynamic focus of the fore part of the aircraft from the center of the masses, which leads to losses on balancing and reduction of fuel efficiency of the aircraft in cruising mode of flight. Because for the current work is considered a cruise flight of the aircraft in the mode of $M \sim 1.5$ let's look at the fore part in the shape of a rotational body.

Thus, it is possible to draw an unequivocal conclusion that the criterion of a choice of rational geometrical parameters of a nose part of an airplane is the minimum value of the aerodynamic resistance coefficient, depending on basically geometrical options. This criterion can be written mathematically as follows (see (2)):

$$
A^{*}=\operatorname{ArgMin}\left(C_{x \text { nose }}\left(\lambda_{\text {nose }}, d, \theta\right)\right) \text {. }
$$

\subsection{Selection of performance evaluation method}

At the present time a significant scientific and technical reserve has been accumulated in the field of research of aerodynamic characteristics of bodies of various shapes and geometry, the main branch institute and center of competence of aerodynamics in the Russian Federation is the Central Aerodynamic Institute named after Professor N.E. Zhukovsky (TsAGI). Based on TsAGI's extensive work to determine the aerodynamic performance of various components of the aircraft, empirical dependencies of the AP on a few geometric parameters were obtained. These methods and dependencies are presented in [7] and are used to determine the AP ( $C_{x}$ and $C_{y}$ ) of the main components of the aircraft (wing, fuselage, plumage) and build integral AP of the aircraft. The empirical approach is widely used by the country's leading aviation and missile companies.

However, because these dependencies have been obtained from numerous wind tunnel experiments on wind tunnel models, there are certain assumptions in the wind tunnel estimates due to model manufacturing tolerances and measurement errors.

The trend formed in the XXI century of constant toughening of requirements to newly developed aircraft complexes (ACs) by the
State Customer, related to ensuring competitive edge over foreign ACs, requires minimization of design reserves in the development of AKs and a more accurate assessment of the AP of both components and the aircraft as a whole.

In recent years, the main trend and element of product life cycle management in the aircraft industry is the digitalization of the life cycle stages in a single information space, including various types of tests [8] and the creation of electronic twins of the aircraft, including aerodynamic, through the application of mathematical simulation of geometry flow by finite element method [9]. The models received by results of similar digital tests are verified and possess necessary sensitivity to change of geometrical characteristics, are shown as algorithm in Figure 2, also allow to receive result with high accuracy and can be parametrized that essentially reduces terms of research.

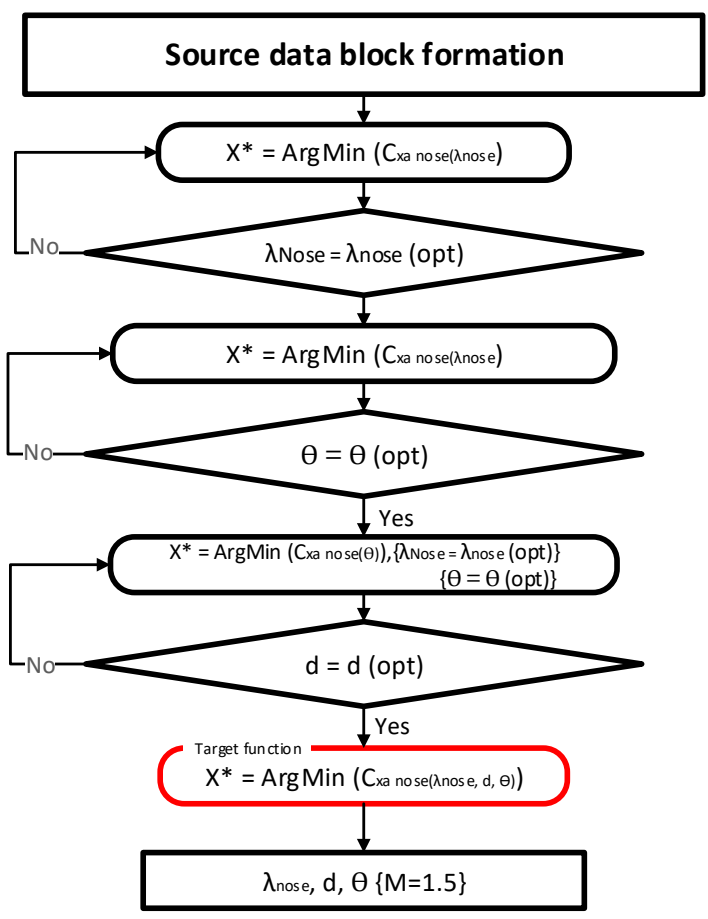

Figure 8: Algorithm for solving the problem of determining the rational parameters of the aircraft fuselage nose section

\subsection{Problem solving algorithm}

For effective solution of the problem within the framework of this work it is necessary to apply methods of mathematical modeling with the use of parametrized mathematical models of the surface, where the main geometrical parameters of the forebody indicated earlier are used as variables of parametrization.

Main calculation parameters are accepted as a block of input data: altitude and speed parameters of flight, values of angles of attack, roll and slide, as well as atmospheric parameters at a selected altitude and speed.

The problem is solved iteratively, and at the first stage is the optimal value of the parameter of elongation of the nose part of the aircraft $\left(\lambda_{\text {nose }}\right)$ as a most comprehensive parameter. Next, for an optimum elongation value, the optimum angle of tangent to the forming curve is selected $(\theta)$ and the final step is to determine the optimal value of the discriminant in the formation curve. $(d)$. 
It should be noted that for each stage a mathematical model of the investigated surface is built, and the range of parameter values can change based on the conditions of ensuring the smoothness of the fore-body surface (absence of double curvature) [10], [11].

\subsection{Selecting the number of settlement cases}

Calculated flight conditions are shown in Table 1. During the calculation the atmospheric parameters are accepted in accordance with the international standard atmosphere.

Table 1: Calculated conditions for solving the problem

\begin{tabular}{|c|c|c|}
\hline & Parameter & Value \\
\hline $\mathbf{1}$ & Estimated number of M flight & $1.5 \mathrm{M}$ \\
\hline $\mathbf{2}$ & Estimated flight altitude & $11000 \mathrm{~m}$ \\
\hline $\mathbf{3}$ & Calculated Flight Angle of Attack $(\alpha)$ & $2^{0}$ \\
\hline $\mathbf{4}$ & Calculated sliding angle $(\beta)$ & $0^{0}$ \\
\hline $\mathbf{5}$ & Calculated roll angle $(\gamma)$ & $0^{0}$ \\
\hline $\mathbf{6}$ & Atmospheric parameters & $\begin{array}{c}\text { In accordance with } \\
\text { the ISA }\end{array}$ \\
\hline
\end{tabular}

The calculation is performed in the selected calculation conditions for each of the values of the parametrization variables and in accordance with the algorithm presented in Part 4. The range of values of the parameterization variables and the step are shown in Table 2.

Table 2: Value range of variables

\begin{tabular}{|c|c|c|c|c|c|c|c|c|}
\hline \multicolumn{8}{|c|}{ Parameterization parameter values } \\
\hline $\min$ & $\max$ & step & $\begin{array}{c}\lambda_{\text {mise }} \\
\mathrm{n}\end{array}$ & $\max$ & step & min & max & step \\
\hline 2 & 5 & $\begin{array}{c}1 \\
(0.5 \text { from } \\
2 \text { to 3) }\end{array}$ & 20 & 45 & 5 & 0.5 & 0.8 & 0.1 \\
\hline
\end{tabular}

The total number of settlement cases for the calculation is determined by formula (3):

$$
N=n_{\lambda}+n_{\theta}+n_{d},
$$

Where $n_{\lambda}$ - fuselage fore-nose extension parameter number, $n_{\theta}$ - number of angle values of the tangent curvature parameter, $n_{d}$ - discriminant parameter number of the constitutive curve.

Thus, to solve the problem it is necessary to make 15 calculation experiments.

In accordance with the research algorithm (see Figure 8), a cycle of computational experiments to determine the dependence of values has been performed in this work $\mathrm{C}_{\mathrm{x} \text { nose }}$ the nose of the aircraft from its extension ( $\left.\lambda_{\text {nose }}\right)$, the results of calculations are recorded in Table 3, the dependence of parameters is shown in Figure 9.

Table 3: Results of the dependency calculation $C_{\text {xnose }}$ on $\lambda_{\text {nose }}$.

\begin{tabular}{|c|c|c|c|c|}
\hline & $\theta$ & $\boldsymbol{d}$ & $\lambda_{\text {nose }}$ & $\mathrm{C}_{\mathrm{x} \text { nose }}$ \\
\hline $\mathbf{1}$ & \multirow{3}{*}{$25^{0}$} & \multirow{3}{*}{0.5} & 2 & 0.21 \\
\hline $\mathbf{2}$ & & 2.5 & 0.15 \\
\hline $\mathbf{3}$ & & 3 & 0.11 \\
\hline $\mathbf{4}$ & \multirow{3}{*}{0} & 4 & 0.06 \\
\hline & & 5 & 0.04 \\
\hline
\end{tabular}

As can be seen from Table 3 and Figure 6, there is an inverse relationship between $\lambda_{\text {nose }}$ and $C_{\text {xnose }}$ for the nose section of the aircraft, so that the body with elongation has minimal aerodynamic resistance $\lambda_{\text {nose }}=5$.

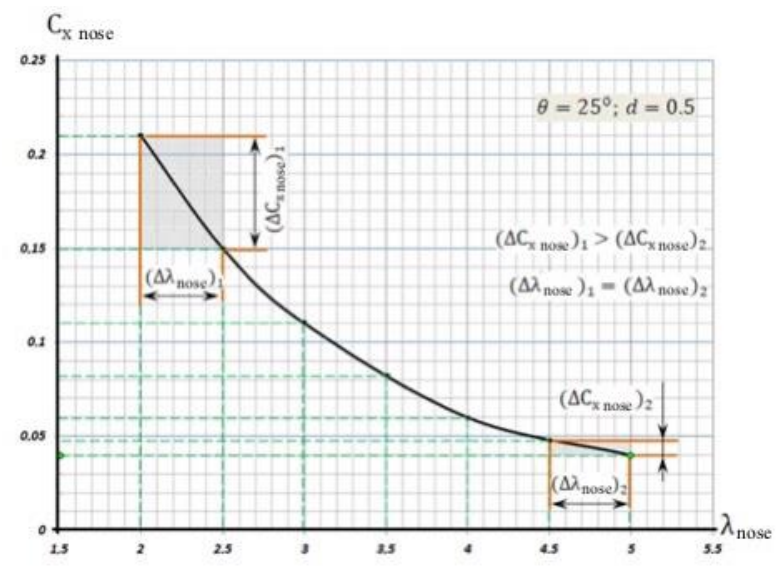

Figure 9: Dependency $C_{\text {xnose }}$ on $\lambda_{\text {nose }}$

Taking into account these results in accordance with the algorithm (see Figure 8), we have estimated the change in the value of $C_{\text {xnose }}$ change angle $\theta$. In this case, to increase the sensitivity of the model to changes in the angle of tangent in the geometric model of the object surface, a spherical blunting of the spout with the $r=15 \mathrm{~mm}$ in this case the influence of the coefficient appears for the model $C_{\text {xattenuation }}$ in case of blunting, which is for $\mathrm{M}=1.5 C_{\text {xattenuation }}=0.68$, and for $r=15 \mathrm{~mm}$ $C_{\text {xattenuation }} \sim 0.22$. The results of calculations are given in Table 4, the dependence is shown in Figure 10.

Table 4: Results of the dependency calculation $\mathrm{C}_{\mathrm{x}}$ nose on $\theta$.

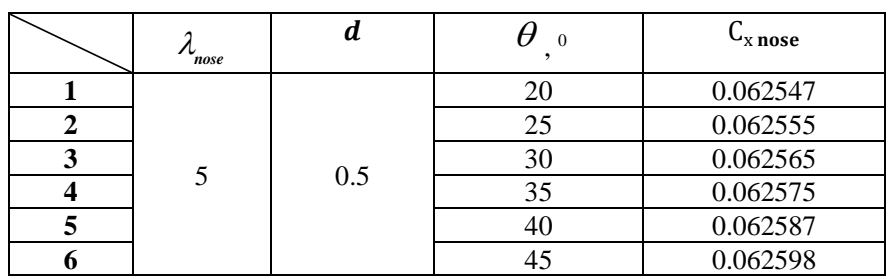

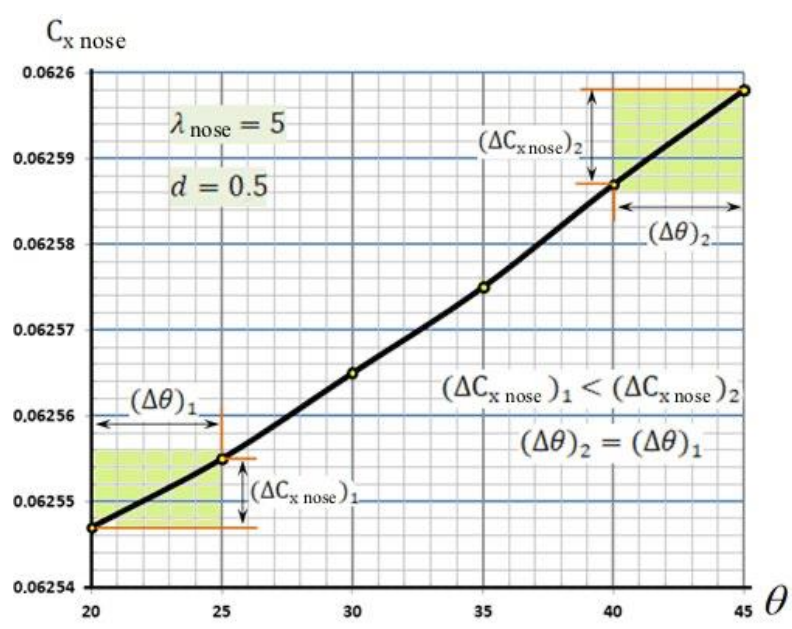




\section{Figure 10: Dependency $\mathrm{C}_{\mathrm{x} \text { nose }}$ at $\theta$}

As can be seen from Table 4 and Figure 10, the aerodynamic resistance of the nose of the aircraft increases when the angle of tangent to the forming nose part of the aircraft. However, the difference between $\mathrm{C}_{\mathrm{x} \text { nose }}$ for $\theta=20^{\mathrm{O}}$ and $\mathrm{C}_{\mathrm{x} \text { nose }}$ for $\theta=45^{\circ}$ at $\lambda_{\text {nose }}=5$ not exceeding $1 \%$. Based on these results, it can be concluded that the angle $\theta$ does not significantly affect the aerodynamic resistance of the nose section of the aircraft at $\mathrm{M}=1.5$. For the next phase of the study, we will accept $\theta=20^{\circ}$ as a parameter with minimal aerodynamic resistance.

The next step in the study of the dependence of aerodynamic characteristics of the nose section of the aircraft on its geometrical parameters is to determine the dependence of $C_{x \text { nose }}$ from the curvature of the constituent $-d$. To determine the effect of curvature on the $\mathrm{C}_{\mathrm{x} \text { nose }}$ aircraft, a comparative analysis of the conical nose AP and the parabolic nose (at $\mathrm{d}=0.5$ ) in determining the dependence of the $C_{x \text { nose }}$ on $\lambda_{\text {nose }}$. The results of this analysis are listed in Table 5, the dependencies are shown in Figure 11.

Table 5: Comparison $\mathrm{C}_{\mathrm{x}}$ nose at $\boldsymbol{d}$.

\begin{tabular}{|c|c|c|c|}
\hline & $\lambda_{\text {nose }}$ & $\mathrm{C}_{\mathrm{x} \text { parabola }}$ & $\mathrm{C}_{\mathrm{x} \text { cone }}$ \\
\hline 1 & 2 & 0.21 & 0.23 \\
\hline 2 & 2.5 & 0.15 & 0.17 \\
\hline 3 & 3 & 0.11 & 0.13 \\
\hline 4 & 4 & 0.06 & 0.08 \\
\hline 5 & 5 & 0.04 & 0.059 \\
\hline
\end{tabular}

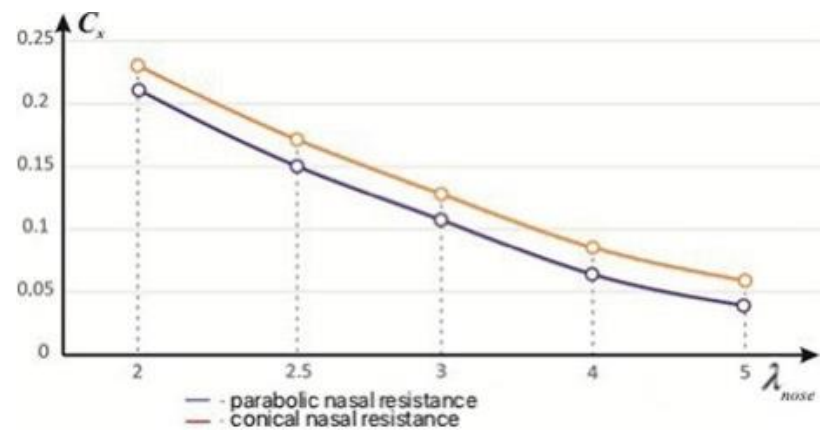

Figure 11: Comparison of nasal AP with conical and parabolic forming parts

As can be seen from Table 4 and Figure 8, both for conical and parabolic forming dependencies obtained in Figure 6 are fair. However, for the parabolic formative level $\mathrm{C}_{\mathrm{x}}$ nose lower than conical for all values $\lambda_{\text {nose }}$. Based on these results, it can be concluded that the nose section of the aircraft with parabolic formation has the lowest aerodynamic resistance.

To determine the rational value of the curvature forming within the framework of this work the dependence analysis has been performed $\mathrm{C}_{\mathrm{x} \text { nose }}$ at $d$ for the nose section with the previously selected rational parameters $\lambda_{\text {nose }}$ and $\theta$, the results of this assessment are shown in Table 5, the dependence is shown in Figure 12.

Table 6: Dependency $\mathrm{C}_{\mathrm{x} \text { nose }}$ at $\boldsymbol{d}$ parabolic forming.

\begin{tabular}{|c|c|c|c|c|}
\hline & $\lambda_{\text {nose }}$ & $\boldsymbol{\theta}$ & $\boldsymbol{d}$ & $\mathrm{C}_{\mathrm{x} \text { nose }}$ \\
\hline 1 & \multirow{2}{*}{5} & $20^{0}$ & 0.5 & 0.062547 \\
\cline { 1 - 3 } & & & 0.6 & 0.062551 \\
\hline
\end{tabular}

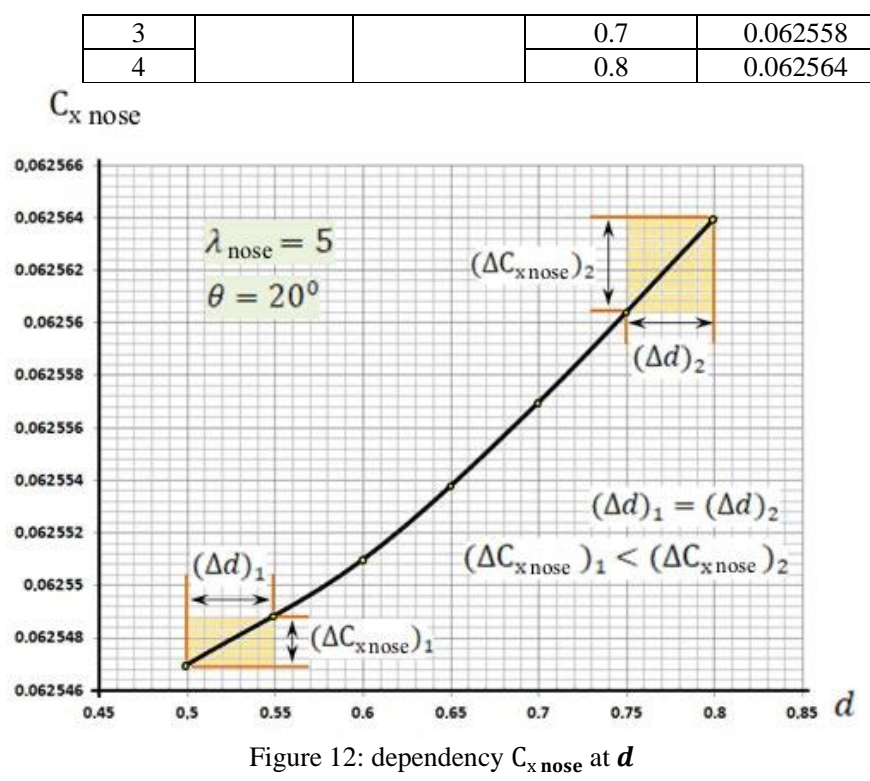

As can be seen from Table 5 and Figure 12, when the curvature of the aircraft nose increases, the aerodynamic resistance of the nose part increases. However, the difference between $C_{x \text { nose }}$ at $d=0.5$ and $\mathrm{C}_{\mathrm{x} \text { nose }}$ for $d=0.8$ at $\lambda_{\text {nose }}=5$ and $\theta=20^{\circ}$ not more than $1 \%$. Based on these results, it can be concluded that parameter $d$ does not significantly affect the aerodynamic resistance of the nose section of the aircraft at $M=1.5$. As a rational value $d$ at $\mathrm{M}=1.5$ take $d=0.5$ as a parameter with minimal aerodynamic resistance.

\subsection{Optimal parameters}

Within the framework of the work performed by the methods of mathematical modeling the dependences of aerodynamic resistance of the nose part of the aircraft on the main geometrical parameters varying in the selected range were obtained for supersonic cruise of the aircraft at $M=1.5$. The following conclusions can be drawn from these studies:

1. Parameter of elongation of the nose of the aircraft $\lambda_{\text {nose }}$ has a significant impact on the aerodynamic characteristics of the nose section of the aircraft. Dependence $C_{x \text { nose }}$ from $\lambda_{\text {nose }}$ is reversed: when the parameter value is increased $\lambda_{\text {nose }}$ level $\mathrm{C}_{\mathrm{x} \text { nose }}$ is decreasing. Reasonable value $\lambda_{\text {nose }}$ to ensure minimum aerodynamic resistance in the selected flight mode is $\lambda_{\text {nose }}=5$.

2. The parameter of the angle of tangent curvature to the forming nose part of the aircraft $\theta$ does not have a significant impact on the aerodynamic characteristics of the nose section of the aircraft when $M=1.5$. Dependency $\mathrm{C}_{\mathrm{x} \text { nose }}$ for $\theta$ is direct: when the parameter value is increased $\theta$ level is increasing $\mathrm{C}_{\mathrm{x} \text { nose }}$. Rational importance $\theta$ to ensure minimum aerodynamic resistance in the selected flight mode is $\theta=20^{\circ}$.

3. The nose section of the aircraft with a parabolic formation has a lower level of aerodynamic resistance than the nose section with a conical formation 3 . 
4. The parameter of curvature of the forming nose part of the aircraft $d$ does not have a significant impact on the aerodynamic characteristics of the nose part of the aircraft at $\mathrm{M}=1.5$. Dependency $\mathrm{C}_{\mathrm{x} \text { nose }}$ from $d$ has a direct character: when the value of parameter $d$ increases, the level $C_{x}$ nose increases. The rational value $\mathrm{d}$ for minimum aerodynamic resistance in the selected flight mode is $d=0.5$.

Thus, based on the conclusions (1-4) can be concluded that the main geometric parameter of the nose of the aircraft, which determines the level of its aerodynamic characteristics, for $\mathrm{M}=$ 1.5 is an extension of the nose of the aircraft $\lambda_{\text {nose }}$.

The nose section of the aircraft with rational geometrical parameters has minimal aerodynamic resistance: $\lambda_{\text {nose }}=5$, $\theta=20^{\circ}, d=0.5$.

\section{Conclusion}

The main conclusion from the materials presented in this paper is that optimization of the development cycles of aircraft components leads to optimization of the product life cycle as a whole. The considered example of forming an optimization basis for choosing rational geometric parameters of RPO clearly showed a significant reduction in the RPO design cycle due to the use of complex techniques.

In this work, a total of about 90 computational experiments were performed, with an average labor intensity (if there is free computing power) of $\sim 5 \mathrm{~h} / \mathrm{h}$. Thus, using the results of this work in the design of a new supersonic aircraft will save $\sim 450 \mathrm{~h} / \mathrm{h}$ of research work, which is $\sim 2.5$ months of work for one specialist of the organization.

The introduction of such an integrated approach in all industries and for all types of products will make it possible to make a new leap in the field of mechanical engineering in the shortest possible time. [12].

\section{References}

[1] E.S. Wentzel, Operation research, Moscow: Science, 1988.

[2] A.V. Barabanov, S. A. Serebryansky, "Approach to determining the rational shape of a supersonic aircraft using mathematical modeling methods", Vestnik SURGU, 2020

[3] V.A. Kaplun, Radar domes, Moscow: Sov. Radio, 1974.

[4] L.G. Artamonova, A.V. Kuznetsov, N.N. Pesetskaya, Verification of aerodynamic characteristics of the aircraft. Moscow, MAI, 2010

[5] G.S. Byushgens, Aerodynamics and flight dynamics of trunk aircraft, TSAGI, 1995.

[6] G.S. Byushgens, Aerodynamics, stability and control of supersonic aircraft, Phismatlit, 1998.

[7] G.A. Kolesnikov, V.K. Markov, A.A. Michailyuk, "Aerodynamics of aircraft". Moscow, Machinostroenie, 1993

[8] A.V. Barabanov, S.A. Serebryansky, "Algorithm for the formation of rational geometric shape of the radio-transparent nose radar fairing of the aircraft," Engineering J.: Science and innovation, 1, 2020, doi: 10.18698/2308-6033-2020-1-1948

[9] S.A. Serebryanskii, A.V. Barabanov, "To the Question of Optimizing Product Life Cycle STAGES," Proc. of 12th International Conference Management of Large-Scale System Development, MLSD, doi: 10.1109/MLSD.2019.8911045, 2019

[10] D.D. Kulikov, V.S. Babanin, "Creating a parametric part model in the CAD system environment", Sci. and Tech. J. of Information Technologies, Mechanics and Optics, 4 (74), 2011.

[11] P.A. Koporushkin, A.S. Partin, D.V. Kurennov, "Algorithm for calculating parametrized objects in the system of geometric modeling of machinebuilding products", Automation and modern technologies, 2008.
[12] S. A. Serebryansky, A.V. Barabanov, "An approach to determining the rational shape of the nose of a supersonic aircraft using mathematical modeling methods", Bulletin of SUSU, mechanical engineering, 20(3), 2020. 